\title{
Tricuspid valve endocarditis following peripheral venous cannulation - An unusual aetiology
}

Kumara D $M^{1}$, Peranantharajah $T^{1}$, Guruparan $M^{1}$, Rajanthi $R^{1}$

${ }^{1}$ Teaching Hospital Jaffna

\begin{abstract}
Isolated tricuspid valve endocarditis is rarely seen without intravenous drug abuse, invasive cardiac procedure or previous valvular disease. Another often overlooked but rarely seen risk factor is peripheral intravenous cannulation. In such cases poor cannula line care and unhygienic practices predispose patients to right sided infective endocarditis. The patient in this case developed tricuspid valve endocarditis following treatment for Dengue Hemorrhagic fever. He had pulmonary involvement as well. Blood culture was positive with MRSA and he was treated with prolonged course of IV antibiotics without surgical interventions. Eventually, he recovered completely and was discharged after 8 weeks of hospital stay.
\end{abstract}

\section{Key words}

Tricuspid valve endocarditis, Right sided infective Endocarditis (RSIE), Peripheral intravenous cannulation

\section{Introduction}

Infective endocarditis is associated with high morbidity and mortality and in the general population Right Sided Infective Endocarditis (RSIE) is less common than left sided infective endocarditis, encompassing only less than $10 \%$. Among RSIE, tricuspid valve is involved in more than $90 \%$ of the cases (1).

The main risk factors for Tricuspid valve Endocarditis are intravenous drug abuse, infection of cardiac implantable devices and indwelling lines, especially chronic IV access used for haemodialysis, parenteral nutrition and chemotherapy. Pleuro pulmonary manifestations are more frequent with RSIE (2). Herein, we present a 19 year old boy with
Tricuspid valve infective endocarditis complicated with bronchopneumonia following peripheral IV cannula site infection. We report this case to highlight the often neglected but preventable cause of RSIE in the developing world.

\section{Case presentation}

A 19 year old previously healthy boy was treated for Dengue Hemorrhagic Fever with hospital stay for seven days. On day 5, he developed pain and swelling at the peripheral cannula site with reappearance of few fever spikes, IV cannula was changed and treated with oral Flucloxacillin.

After one week of discharge, he presented to the Emergency Unit with fever with chills and rigors associated with productive cough, hemoptysis and difficulty in breathing for 2 days. He denied any history of intravenous drug abuse, sexual promiscuity or smoking but he consumed alcoholic beverages occasionally.

On presentation, the patient was ill looking, febrile and tahcypnoeic with respiratory rate of 36 breaths/min and $\mathrm{SPO}_{2}$ of $87 \%$ on air. He was tachycardic and blood pressure was $100 / 70 \mathrm{mmHg}$. A physical examination showed bilateral crackles on auscultation. There was no audible heart murmur. He didn't have any peripheral stigmata if infective endocarditis.

The patient's laboratory data revealed the following: high white cell count with mild anemia, mild thrombocytopenia and very high inflammatory markers. Renal function test was normal and liver enzymes were slightly elevated. HIV, VDRL, Hepatitis B and C screening were negative. An initial chest radiograph revealed bilateral alveolar infiltrates. Blood cultures were taken and he was started on broad spectrum antibiotics. 


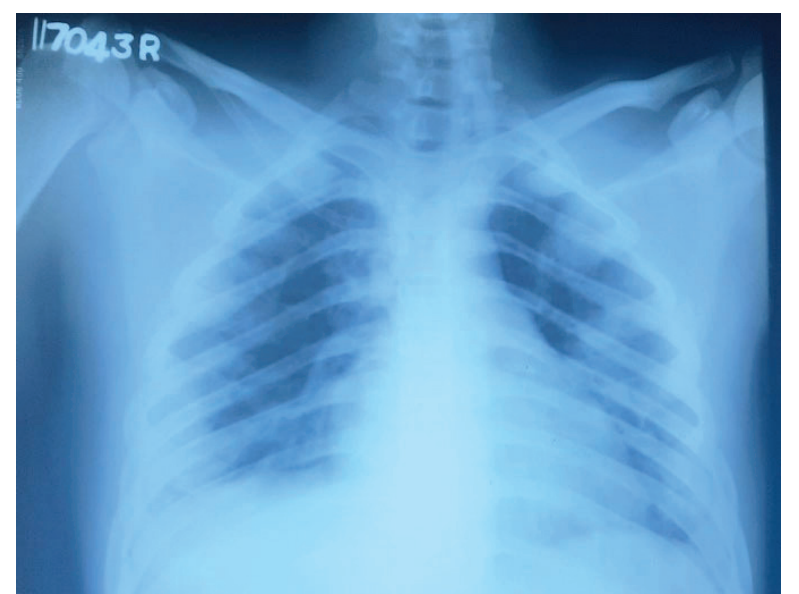

Figure 1:- Chest Xray of the patient.

As clinical improvement was unsatisfactory a Trans Thoracic Echocardiogram (TTE) was arranged and it showed an oscillating mass sizing $23 \mathrm{~mm}$, attached to septal leaf of tricuspid valve, favoring the diagnosis of Tricuspid valve endocarditis. LVEF was $70 \%$ and other valves were normal. There were no intracardiac shunts. Blood culture was positive for MRSA.

Considering the size of the vegetation, Surgical opinion was sought, but was decided to consider that option only if medical treatment fails. However, he fully recovered with 6 week course of antibiotic therapy intravenously, IV Vancomycin initially followed by IV Teicoplanin as he developed mild renal impairment while on Vancomycin. He was discharged after 50 days of hospital stay and the follow up TTE in one month time was completely normal without vegetation.

\section{Discussion}

Right sided infective Endocarditis demonstrates with fewer cardiac symptoms and fewer classic signs of infective endocarditis such as the presence of murmur and peripheral cutaneous manifestations (3). Additionally, the sensitivity and specificity of modified Duke's criteria in RSIE has not been studied. RSIE is often found in intravenous drug users and patients with indwelling central venous catheters but in our patient attributed risk factor is recent intravenous cannulation. This is not an uncommon risk factor in developing countries but ignored in literature (4). It is prominent in these countries where intravenous drug abuse is relatively lower (4).

RSIE always has a better outcome than left sided infective endocarditis (5). Staphylococcus aureus, a virulent organism to valves is the most common organism implicated in RSIE and causes more severe disease with persistent sepsis with multiorgan dysfunction and higher mortality.

Uncomplicated infective endocarditis is predicted by low risk features like good response to therapy, MSSA(Methicillin Sensitive Staph. aureus), small vegetation size $(<20 \mathrm{~mm})$, no features of peripheral embolization, absence of metastatic infection, lack of involvement of left sides valves/prosthesis, absence of severe immunocompromised state and the absence of right heart failure features induced by tricuspid valve regurgitation. These patients usually respond well to 2 week treatment regime and don't need surgery (6). In complicated cases, 4-6 week therapy with IV antibiotics is needed and they might need surgery too.

The goals of surgery are to eradicate the infection and achieve hemodynamic correction with adequate strategies and the surgery can yield good results.

\section{Conclusion}

The development of Cardiology practices including Cardiac surgeries and availability of rational antibiotics have improved the outcome of infective endocarditis, however preventable bad clinical practices such as ill - timed poorly cared and unnecessary intravenous lines incidentally trigger more cases of Tricuspid valve endocarditis. Early Multi-disciplinary approach involving Microbiologist, Cardiologists and Cardio thoracic Surgeons will lead to better outcome in these patients. 


\section{References}

1. Murdoch DR, Corey GR, Hoen B, et al. Clinical presentation, etiology, and outcome of infective endocarditis in the 21 st century: the International Collaboration on EndocarditisProspective Cohort Study. Arch Intern Med 2009; 169:463-73

2. Demin A.A, Drobysheve V.P, Welter O.Y, Pulmonary complications of infective endocarditis in intravenous drug users.

3. FronteraJA, Gradon JD. Right-side infective endocarditis in injection drug users: review of proposed mechanisms of pathogenesis. Clin Infect Dis 2000;30:374-9.
4. Godsent Isiguzo, Collins Ugwu, Uma Kalu, Ndudi Obeka, Basil Ezeokpo Right sided endocarditis secondary to prolonged intravenous cannulation Am J Respir Crit Care Med 193;2016:A7013

5. Robbins MJ, Frater RW, Soeiro R, et al. Influence of vegetation size on clinical outcome of right-sided infective endocarditis. The American Journal of Medicine. 1986;80:165

6. Ribera E, Gomez-Jimenez J, Cortes E, del Valle O, Planes A, et al. Effectiveness of cloxacillin with and without gentamicin in short-term therapy for right-sided Staphylococcus aureus endocarditis. 1996 Dec 15;125(12):969-74. 